\title{
Nanoparticle Formation of Puerarin- $\beta$-Cyclodextrin Inclusion Complex Using SEDS: Dissolution Enhancement
}

\author{
H. P. LEI, K. R. ZHANG ${ }^{1}$, J. WANG ${ }^{1}$, H. ZHANG, Q. L. SHI ${ }^{1}$, F. H. GE ${ }^{1 *}$ AND Q. B. HAN ${ }^{2}$
}

School of Pharmaceutical Sciences, Xiangnan University, Chenzhou 423000, ${ }^{1}$ School of Pharmaceutical Sciences, Sun Yat-Sen University, Guangzhou 510006, PR China, ${ }^{2}$ School of Chinese Medicine, Hong Kong Baptist University, 999077, Hong Kong

\section{Lei et al.: Puerarin- $\beta$-Cyclodextrin Nanoparticle by Supercritical Fluids}

Inclusion complex nanoparticles of puerarin and $\beta$-cyclodextrin were prepared using solution-enhanced dispersion by supercritical fluids to improve the dissolution rate of puerarin. The factors that influenced particle size and inclusion yield such as, pressure, temperature, flow rate of $\mathrm{CO}_{2}$, and flow rate of the solution, were investigated. Scanning electron microscopy, $X$-ray diffraction and Fourier-transform infrared spectroscopy were used to characterize the products. Release behaviour of inclusion complex nanoparticles of puerarin was also studied. Elevated temperatures increased the inclusion yield. Elevated pressures reduced the particle size. Scanning electron microscopy, X-ray diffraction and Fourier-transform infrared spectroscopy confirmed the formation of inclusion complex nanoparticles of puerarin. The accumulated release rate of inclusion complex nanoparticles of puerarin reached $98 \%$ within 5 min, markedly higher than that of the puerarin powder and its physical mixture. Inclusion complex nanoparticles of puerarin prepared by solution-enhanced dispersion by supercritical fluids can greatly improve the in vitro release of puerarin.

Key words: Supercritical $\mathrm{CO}_{2}$, inclusion complex, nanoparticle, puerarin

Puerarin is the major bioactive constituent isolated from the root of the Pueraria lobata (Willd.) Ohwi, known as Gegen (Chinese) in traditional Chinese medicine. Puerarin has a wide spectrum of pharmacological properties such as cardioprotection, vasodilation, neuroprotection, antioxidant, antiinflammation, anticancer, alleviating pain, promoting bone formation, attenuating insulin resistance, and inhibiting alcohol intake ${ }^{[1]}$. However, due to the polyphenol ring and polyhydroxy groups in puerarin structure its water- and fat-solubility is low. It is also insoluble in chloroform, difficult to be absorbed through the gastrointestinal tract, has low oral bioavailability, which limits the clinical application of puerarin. At present, there is only one dosage form, the puerarin injection is in clinic ${ }^{[2]}$. After oral administration in adults, $0.78 \%$ of puerarin is excreted in urine after $36 \mathrm{~h}$, and $73.3 \%$ is excreted in feces after $72 \mathrm{~h}^{[3]}$. This revealed that puerarin is not highly absorbed after oral administration because of its poor solubility. To improve the bioavailability of puerarin, an inclusion complex of puerarin- $\beta$-cyclodextrin $(\beta-C D)$ is a good alternative.
CDs are torus-like molecules that have a hydrophilic outer surface and a lipophilic internal cavity, into which suitable molecules may enter to form an inclusion complex. Several methods, such as grinding, co-precipitation, spray drying, and freeze drying, have been developed to form an inclusion complex ${ }^{[2,4]}$. Unfortunately, many of these methods have shortcomings, including low efficiency, long processing times, high levels of residual organic solvent, and the need for an additional drying step. Over the last few years, the employment of supercritical $\mathrm{CO}_{2}$ as an abnormal inclusion medium has received increasing interest ${ }^{[4-10]}$. Supercritical $\mathrm{CO}_{2}$ antisolvent technology (SAS) has proven to be a convenient, product-controlled, and environment-friendly method for preparing inclusion complex nanoparticles

This is an open access article distributed under the terms of the Creative Commons Attribution-NonCommercial-ShareAlike 3.0 License, which allows others to remix, tweak, and build upon the work non-commercially, as long as the author is credited and the new creations are licensed under the identical terms

Accepted 11 May 2019

Revised 03 January 2019

Received 04 September 2018

Indian J Pharm Sci 2019;81(4):601-607 
$(\mathrm{ICN})^{[11]}$. Among the many specific methods that have been developed ${ }^{[12]}$, solution-enhanced dispersion by supercritical fluids (SEDS) is a particularly efficient method for producing ICN because of its specially designed nozzle ${ }^{[13,14]}$.

In the present work, SEDS was used to prepare ICN of puerarin (ICNP) and improve the dissolution properties of puerarin. In the experiments reported herein, the effects of pressure, temperature, $\mathrm{CO}_{2}$ flow rate, and solution flow rate were investigated and the ICNP was characterized by scanning electron microscopy (SEM), X-ray diffraction (XRD), Fourier-transform infrared (FTIR) spectroscopy and dissolution testing.

\section{MATERIALS AND METHODS}

Puerarin (98.5\% purity) was supplied by the Xi'an Shengqing Biotechnology Limited Company (Shanxi Province, China) and $\beta-C D$ was obtained from the Shandong Binzhou Zhiyuan Bio-Technology Co., Ltd. (Shandong Province, China). High-purity $\mathrm{CO}_{2}$ $(99.99 \%)$ was purchased from the Guangzhou Gas Factory Co., Ltd. (Guangdong, China). All other chemicals were of analytical grade.

\section{Preparation of ICNP using SEDS:}

The SEDS apparatus used in this study is the same as that described previously ${ }^{[15]}$. The experimental apparatus used for preparing the ICNP is primarily made up of a high-pressure plunge pump for delivering $\mathrm{CO}_{2}$, a high-pressure constant flow pump for liquid feeding, and a windowed precipitation chamber of $150 \mathrm{ml}$. A typical experiment was carried out as follows: supercritical $\mathrm{CO}_{2}$ was added to the precipitator vessel until the preset pressure and temperature were achieved, and these were maintained. The puerarin- $\beta-C D$ solution was prepared by dissolving puerarin and $\beta-C D$ (1:1 molar ratio) in DMSO. The puerarin solution was then injected at a flow rate until predetermined quantity of the solution had been processed. Supercritical $\mathrm{CO}_{2}$ was allowed to flow continuously for $20 \mathrm{~min}$ to eliminate the remaining organic solvent from the particles. Finally, the prepared particles were collected on a stainless-steel filter screen after depressurization.

The experiment was carried out at pressures of 10 $20 \mathrm{MPa}$ and temperatures of $35-55^{\circ}$. The nozzle diameter was $0.12 \mathrm{~mm}$. The concentrations of puerarin and $\beta$-CD in DMSO solution were 10.40 and $28.40 \mathrm{mg} / \mathrm{ml}$, respectively. The flow rates of the solution and supercritical $\mathrm{CO}_{2}$ were $0.5-1.5 \mathrm{ml} / \mathrm{min}$ at the pump and 3-9 1/h, respectively. Tables 1 and 2 show the detailed experimental conditions.

\section{Preparation of physical mixture:}

A physical mixture was prepared by grinding the puerarin and $\beta-C D$ powders with 1:1 molar ratio in a mortar with a pestle. After preparation, the puerarin, $\beta$-CD physical mixture was used promptly to protect against absorption of moisture from the air.

\section{Determination of the inclusion yield:}

The inclusion yield was measured with the same differential solubility method ${ }^{[16]}$ that has been previously applied to measure the included/free drug ratio in piroxicam/CD inclusion complex, since the method is readily extended to other drug/CD systems ${ }^{[4,17]}$. This method is based on the differing solubility of the drug and $\mathrm{CD}$ in acetonitrile. When acetonitrile is mixed with the treated sample, the non-included drug is soluble, while the included drug remains trapped. The amount of the free drug can thus be measured. If the mixture of water-acetonitrile $(1: 1 \mathrm{v} / \mathrm{v})$ is used instead of pure acetonitrile, the $\mathrm{CD}$ also becomes dissolved and the complex is completely dissolved, allowing the total amount of the drug to be measured ${ }^{[4]}$. The inclusion yield $(\eta)$ can then be measured using the following Eqn. $1, \quad \eta=100 \times(1-$ free puerarin content/total puerarin content). Samples were determined for the free and total drug amount using a spectrophotometer (UV-2600, Shimadzu Corporation, Japan) at $250 \mathrm{~nm}$.

\section{Determination of particle size:}

A sample of $5 \mathrm{mg}$ was dispersed with $5 \mathrm{ml}$ water, and treated with ultrasound of $28 \mathrm{kHz}$ for $1 \mathrm{~min}$. Then the particle size of the sample was determined using a nanometer particle size and zeta potentiometric analyzer (Nano-ZS, Malvern Instruments Ltd., UK). This instrument can measure particle sizes ranging from $0.3 \mathrm{~nm}$ to $10 \mu \mathrm{m}$.

\section{Scanning electron microscopy (SEM):}

Micrographs were obtained with a SEM (Quanta 400, FEI Company, USA). Samples were first coated with gold using a sputter system (E5100, Bio-Rad, München, Germany).

\section{X-ray diffraction (XRD):}

The powder XRD patterns were obtained with an X-ray diffractometer (Empyrean, Panalytical Company, Holland). The patterns were typically gathered over the $2 \theta$ range of $5-45^{\circ}$. 


\section{FTIR spectroscopy:}

FTIR transmission spectra were obtained by a FTIR spectrophotometer (Avatar360, Nicolet Instrument Corporation, USA). The samples were ground and mixed thoroughly with $\mathrm{KBr}$. The $\mathrm{KBr}$ disks were prepared by means of a hydrostatic press. The scanning range was $400-4000 \mathrm{~cm}^{-1}$, and the resolution was $4 \mathrm{~cm}^{-1}$.

\section{Drug release studies:}

Drug release determination was carried out at $37^{\circ}$ for untreated puerarin, its unprocessed physical mixture with $\beta-\mathrm{CD}$, and the ICNP, employing a test described in the Chinese Pharmacopoeia (volume IV, general rule 0931 (2015)). The apparatus was paddle. The dissolution tests were conducted in a vessel containing $900 \mathrm{ml}$ of distilled water with rotation at $50 \mathrm{rpm}$. The sample $(250 \mathrm{mg})$ was added to the chamber and 3-ml aliquots of the dissolution fluid were taken at 1 , $3,5,10,15,20,30,45$, and $60 \mathrm{~min}$. These aliquots were filtered through a membrane (Sarstedt, pore size $0.45 \mu \mathrm{m})$. Each aliquot was replaced with an equal volume of fresh medium. The filtrates were determined spectrophotometrically at $250 \mathrm{~nm}$.

\section{RESULTS AND DISCUSSION}

As shown in Table 1, with a constant solution flow rate of $1 \mathrm{ml} / \mathrm{min}$ and $\mathrm{CO}_{2}$ flow rate of $61 / \mathrm{h}$, under different pressures and temperatures, the inclusion yield of ICNP prepared using SEDS ranged from 80.49 to $98.23 \%$. Overall, the inclusion yield was higher at a higher temperature $\left(55^{\circ}\right)$ because of a shift in the equilibrium of complexation toward the complexed form $^{[18]}$. This agreed with the finding of Banchero and Manna $^{[4]}$.

In Table 2, with the temperature and pressure fixed at $45^{\circ}$ and $15 \mathrm{MPa}$, respectively, under different solution flow rates and $\mathrm{CO}_{2}$ flow rates, the inclusion yield of ICNP prepared using SEDS ranged from 76.48 to $92.76 \%$. When the solution flow rate was $0.5 \mathrm{ml} / \mathrm{min}$, the inclusion yield increased with the increasing $\mathrm{CO}_{2}$ flow rate. However, when the solution flow rates were $1 \mathrm{ml} / \mathrm{min}$ and $1.5 \mathrm{ml} / \mathrm{min}$, respectively, the inclusion yield decreased with the increasing $\mathrm{CO}_{2}$ flow rate.

Table 1 show that the particle size of ICNP decreased along with increasing pressures at different temperatures. This result confirmed previous experimental findings ${ }^{[15,19]}$. At lower pressures, the

TABLE 1: INCLUSION YIELD AND PARTICLE SIZE OF INCLUSION COMPLEX NANOPARTICLES UNDER DIFFERENT TEMPERATURES AND PRESSURES

\begin{tabular}{lcccc}
\hline Sample no. & Temperature $\left(^{\circ}\right)$ & Pressure $(\mathrm{MPa})$ & Inclusion yield, $\boldsymbol{\eta}(\%)$ & particle size $(\mathrm{nm})$ \\
\hline 1 & 35 & 10 & $80.4 \pm 93.26$ & $956.7 \pm 90.2$ \\
2 & 35 & 15 & $86.99 \pm 5.12$ & $875.7 \pm 82.4$ \\
3 & 35 & 20 & $87.32 \pm 4.89$ & $701.3 \pm 70.5$ \\
4 & 45 & 10 & $84.25 \pm 4.56$ & $1278.8 \pm 113.5$ \\
5 & 45 & 15 & $82.94 \pm 5.23$ & $684.9 \pm 75.6$ \\
6 & 20 & $82.61 \pm 5.34$ & $643.1 \pm 68.7$ \\
7 & 45 & 10 & $94.21 \pm 6.28$ & $1191.0 \pm 123.8$ \\
8 & 55 & 15 & $98.23 \pm 7.24$ & $706.9 \pm 65.8$ \\
9 & 55 & 20 & $87.96 \pm 5.89$ & $563.3 \pm 54.6$ \\
\hline
\end{tabular}

TABLE 2: INCLUSION YIELD AND PARTICLE SIZE OF INCLUSION COMPLEX NANOPARTICLES UNDER DIFFERENT FLOW RATES OF SOLUTION AND $\mathrm{CO}_{2}$ AT $45^{\circ}$ AND $15 \mathrm{MPa}$

\begin{tabular}{lcccc}
\hline Sample no. & Solution flow rate $(\mathrm{ml} / \mathrm{min})$ & $\mathrm{CO}_{2}$ flow rate $(\mathrm{l} / \mathrm{h})$ & Inclusion yield, $\boldsymbol{\eta}(\%)$ & Particle size $(\mathrm{nm})$ \\
\hline 10 & 0.5 & 3 & $76.48 \pm 2.89$ & $445.2 \pm 34.5$ \\
11 & 0.5 & 6 & $82.01 \pm 4.56$ & $662.7 \pm 52.6$ \\
12 & 0.5 & 9 & $83.32 \pm 4.89$ & $896.1 \pm 82.4$ \\
13 & 1 & 3 & $86.38 \pm 5.34$ & $1256.9 \pm 124.6$ \\
14 & 1 & 6 & $82.94 \pm 4.67$ & $701.3 \pm 66.8$ \\
15 & 1 & 9 & $75.50 \pm 2.56$ & $1127.8 \pm 106.4$ \\
16 & 1.5 & 3 & $92.76 \pm 6.12$ & $1050.1 \pm 95.4$ \\
17 & 1.5 & 6 & $79.08 \pm 3.24$ & $1232.6 \pm 108.6$ \\
18 & 1.5 & 9 & $75.79 \pm 2.15$ & $1126.7 \pm 95.7$ \\
\hline
\end{tabular}

Solution flow rate: $1 \mathrm{ml} / \mathrm{min}$ and the carbon dioxide flow rate: $6 \mathrm{l} / \mathrm{h}(\mathrm{n}=3)$ 
system was closer to the mixture critical point ${ }^{[20]}$, and the mass transfer between the organic solvent and $\mathrm{CO}_{2}$ was slow, causing a gradient change among the local supersaturation states within the mixing region, which resulted in larger particles ${ }^{[21]}$. In contrast, at higher pressures (far above the mixture critical point), the mass transfer was very fast, avoiding the gradient difference within the local supersaturation states, resulting in smaller particles ${ }^{[15]}$.

As shown in Table 1, the relationship between particle size and temperature was complex. Under constant conditions, the density of supercritical $\mathrm{CO}_{2}$ decreased along with increasing temperature, which resulted in smaller mass transfer efficiency from $\mathrm{CO}_{2}$ to solvent and larger particles. Meanwhile, with increasing temperature, the kinetic energy of the solute molecule increased. Small particles collided and aggregated more often, resulting in larger particles. Further, with increasing temperature, the increasing kinetic energy of the solvent molecule resulted in an increasing diffuse velocity of the solvent and smaller particles ${ }^{[22]}$. Finally, with increasing temperature, the viscosity of the solution reduced, which strengthened the atomization effect of the solution, forming smaller particles. Thus it can be seen that the final effect of temperature depended on several impact directions.
In this study, the effects of solution flow rate and $\mathrm{CO}_{2}$ flow rate on particle formation were investigated. As shown in Table 2, with pressure and temperature fixed at $15 \mathrm{MPa}$ and $45^{\circ}$, respectively, the particle enlarged with increasing solution flow rate at various $\mathrm{CO}_{2}$ flow rates. It has been commonly reported that the particle size changes with the solution flow rate in the SAS process $^{[23]}$. The mechanism could be explained by the classical theory of the crystal nucleus growing. When the solution jets into supercritical $\mathrm{CO}_{2}$ through the nozzle, the solute leads to supersaturation and precipitates. For the solution with a high flow rate, there are enough solute molecules for the crystal nucleus to grow, forming larger particles. In contrast, for the solution with a low flow rate, there are few solute molecules for the crystal nucleus to grow, forming smaller particles.

Table 2 shows that at the same solution flow rate the relationship between $\mathrm{CO}_{2}$ flow rate and particle size was complex. With a greater $\mathrm{CO}_{2}$ flow rate, the disturbance of $\mathrm{CO}_{2}$ in the coaxial nozzle is greater. Solute molecules collide violently, resulting in larger particles. Meanwhile, with a greater $\mathrm{CO}_{2}$ flow rate, the effect of SAS is greater. The solute leads to supersaturation, with instant nucleation increasing, resulting in smaller particles.

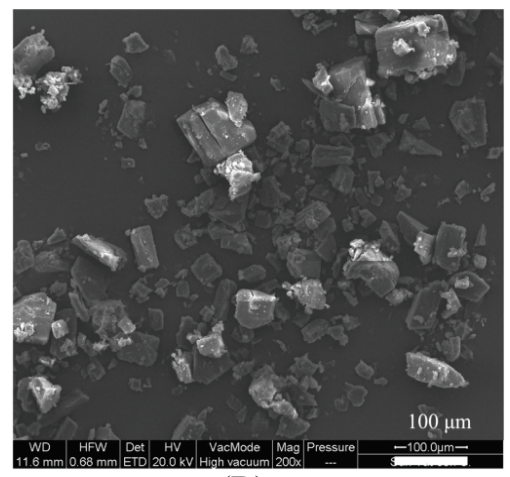

(B)

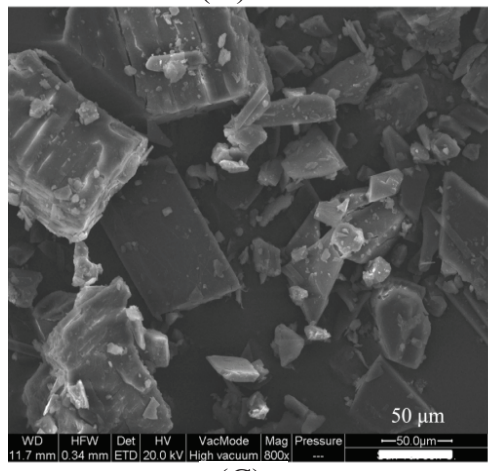

(C)
(D)

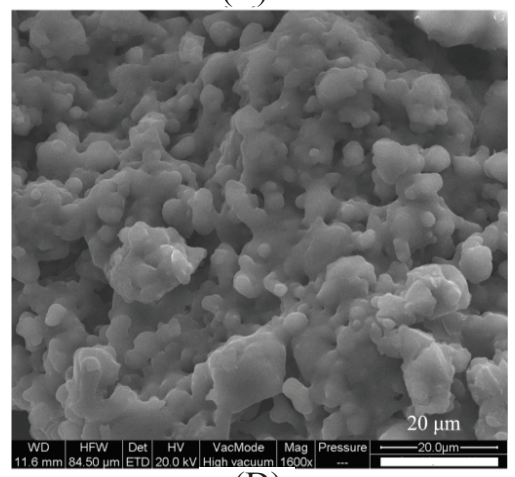

Fig. 1: SEM images

(A) Unprocessed puerarin, (B) unprocessed $\beta-C D,(C)$ an unprocessed puerarin/ $\beta-C D$ physical mixture: 1:1 molar ratio and (D) puerarin/ $\beta-C D$ inclusion complex prepared by SEDS process: 1:1 molar ratio 
In this study, untreated puerarin, $\beta-C D$, their unprocessed physical mixture, and ICNP were analyzed by SEM (fig. 1). Fig. 1 shows that the raw puerarin was asymmetrically bulky. The raw $\beta-C D$ was irregularly clubbed. Their unprocessed physical mixture was asymmetrically nubby and clubbed. The ICNP comprised sticky spherical particles.

Further supporting evidence for the formation of an inclusion complex between puerarin and $\beta-\mathrm{CD}$ was obtained from the powder XRD patterns. The XRD patterns in fig. 2 show that the untreated puerarin and $\beta-C D$ exhibit a series of intense peaks corresponding to their crystallinity. Moreover, the pattern of the physical mixture presents peaks similar to the superposition of the patterns of the untreated puerarin and $\beta-C D$, indicating there was no new substance forming. By comparison, the pattern generated by the ICNP was not similar to those mentioned above; there were no intense peaks of puerarin, and it was not similar to the diffraction curve of $\beta$-CD. It showed that a new solid phase generated, which indicated formation of an inclusion complex.

FTIR spectroscopy was used to obtain further information on the state of the drug in the inclusion complex formulation. Changes in the FTIR spectra such as shifts in characteristic peaks, reduction of or disappearance in peak intensity, and the appearance of new peaks might be connected to drug-CD interactions $^{[24]}$. Fig. 3 showed the FTIR spectra of the puerarin, $\beta-C D$, puerarin $/ \beta-C D$ physical mixture and puerarin $/ \beta$-CD inclusion complex prepared by SEDS. As shown in fig. 3, the IR spectra of the physical mixture were similar to the superposition of the spectra

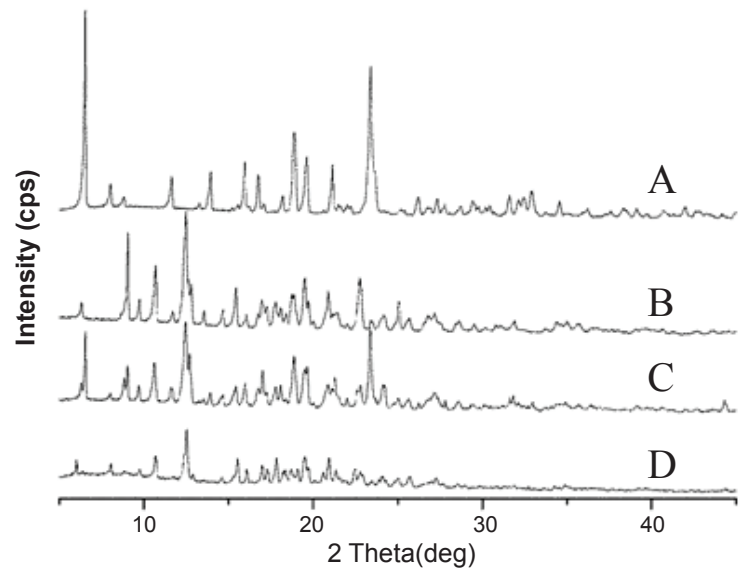

Fig. 2: XRD patterns

(A) Puerarin, (B) $\beta$-CD, (C) puerarin/ $\beta-C D$ physical mixture: 1:1 molar ratio and (D) puerarin/ $\beta-C D$ inclusion complex prepared by SEDS process: 1:1 molar ratio

July-August 2019

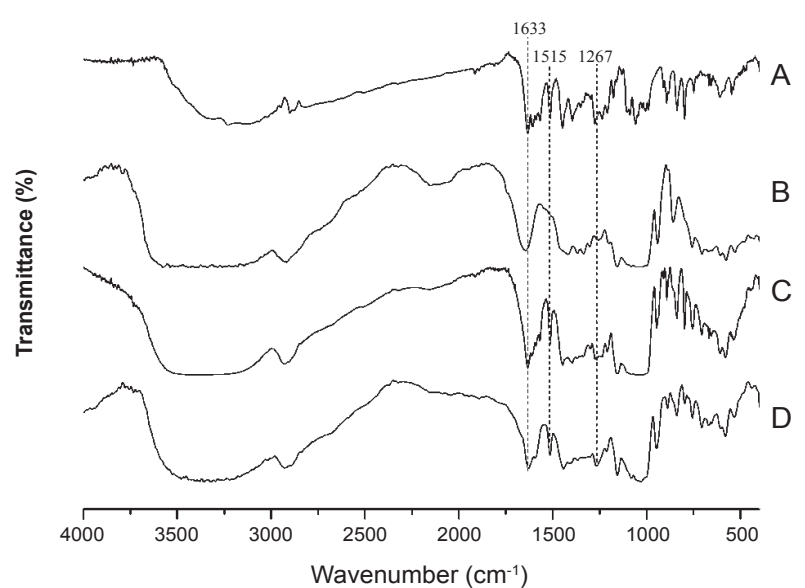

Fig. 3: FTIR spectra

(A) Puerarin, (B) $\beta$-CD, (C) puerarin/ $\beta-C D$ physical mixture: 1:1 molar ratio and (D) puerarin/ $\beta-C D$ inclusion complex prepared by SEDS process: 1:1 molar ratio

of puerarin and $\beta-\mathrm{CD}$, which indicated there was no new substance forming. The IR spectrum of ICNP was similar to that of $\beta-C D$ and the physical mixture, which indicated that the main structures between ICNP and $\beta$-CD were the same. There were some obvious changes of characteristic absorption peaks of puerarin: (1) $\mathrm{C}=\mathrm{O}\left(1633 \mathrm{~cm}^{-1}\right)$ shifted to $1627 \mathrm{~cm}^{-1}$, and (2) the intensity of the benzene ring peak $\left(1515 \mathrm{~cm}^{-1}\right)$ decreased. These changes showed that some puerarin molecules were included in the hydrophobic cavity of $\beta-\mathrm{CD}^{[25]}$. (3) $\mathrm{C}-\mathrm{O}-\mathrm{H}$ peaks at $1267 \mathrm{~cm}^{-1}$ in inclusion complexes had much higher intensity than that of $\beta$-CD. This is evidence of the formation of hydrogen bonds between the hydroxyl groups of $\beta-\mathrm{CD}$ and the carbonyl groups of puerarin. Based on the discussion above, it is reasonable to conclude that the host-guest inclusion complex was formed ${ }^{[26]}$.

Drug release studies were performed in distilled water to evaluate the extent to which the loading process improved the water solubility of puerarin. Fig. 4 shows that in the same experimental conditions, after 5 min the dissolved drug for raw puerarin, the physical mixture and ICNP were 68.69, 78.70 and $98.60 \%$, respectively. The untreated puerarin dissolved slowly. The addition of $\beta$-CD evidently had a positive effect because the untreated physical mixture exhibited higher dissolution rates than the untreated puerarin. The dissolution of ICNP enhanced greatly with 98.60 wt. \% dissolved after $5 \mathrm{~min}$. The ICNP prepared using SEDS improved the solubility and dissolution rate of puerarin.

Uniform spherical ICNP were prepared using SEDS in this study. The procedure parameters such as pressure, temperature, $\mathrm{CO}_{2}$ flow rate, and solution flow rate 


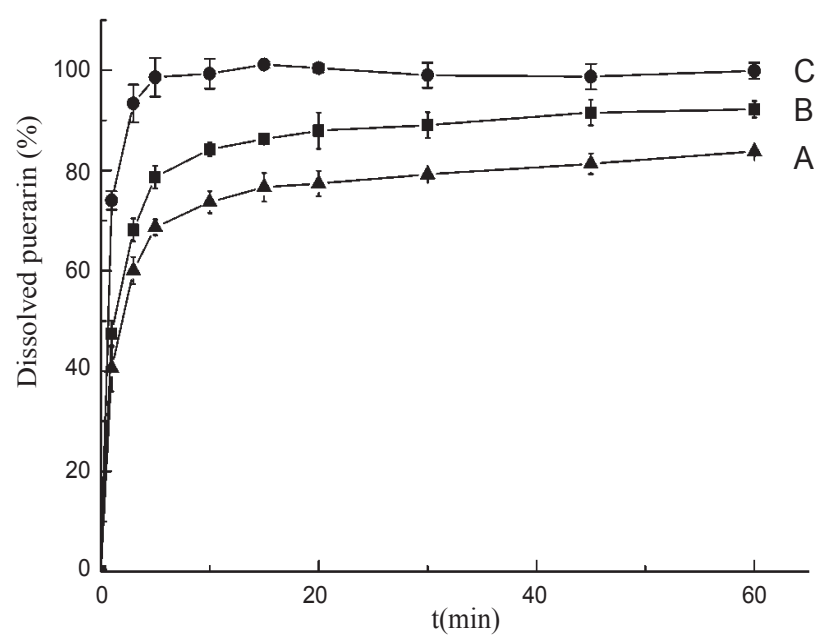

Fig. 4: Dissolution profiles

(A) Unprocessed puerarin, (B) an unprocessed physical mixture: 1:1 molar ratio and $(C)$ puerarin/ $\beta-C D$ inclusion complex prepared by SEDS process: 1:1 molar ratio $(n=3$, mean \pm SD)

were investigated. Elevated temperatures increased the inclusion yield. Elevated pressures reduced the particle size. SEM, XRD and FTIR confirmed the formation of ICNP. The ICNP could enhance the release rate of puerarin, with a quick release effect.

\section{Acknowledgements:}

This work was supported by The Natural Science Foundation of Hunan Province (grant number 20015JJ3111) and Ministry of Health of Hunan Province (grant number B2016092).

\section{REFERENCES}

1. Zhou Y, Zhang H, Peng C. Puerarin: A review of pharmacological effects. Phytother Res 2014;28(7):961-75.

2. Xie J, Yang Y, Shi X, Zhu X, Su W, Wang P. Improvement in solubility and bioavailability of puerarin by mechanochemical preparation. Drug Dev Ind Pharm 2013;39(6):826-35.

3. Zhu X, Su C, Li Z, Yue T, Yan X, Wei H. The metabolic fate of the effective components of puerariae. Acta Pharm Sin 1979;14(3):129-34.

4. Banchero M, Manna L. The use of lysine to enhance the supercritical complexation of ketoprofen and cyclodextrins. J Supercrit Fluid 2012;67:76-83.

5. Naylor A, Lewis AL, Illum L. Supercritical fluid-mediated methods to encapsulate drugs: recent advances and new opportunities. Ther Deliv 2011;2(12):1551-65.

6. Hussein K, Türk M, Wahl MA. Drug loading into $\beta$-cyclodextrin granules using a supercritical fluid process for improved drug dissolution. Eur J Pharm Sci 2008;33(3):306-12.

7. Zhou R, Wang F, Guo Z, Zhao YL. Preparation and characterization of resveratrol/hydroxypropyl- $\beta$-cyclodextrin inclusion complex using supercritical antisolvent technology. J Food Process Eng 2012;35(4):677-86.

8. Lee CW, Kim SJ, Youn YS, Widjojokusumo E, Lee YH,
Kim J, et al. Preparation of bitter taste masked cetirizine dihydrochlori/ $\beta$-decyclodextrin inclusion complex by supercritical antisolvent (SAS) process. J Supercrit Fluid 2010;55(1):348-57.

9. Türk M, Upper G, Steurenthaler M, Hussein KH, Wahl MA. Complex formation of Ibuprofen and $\beta$-Cyclodextrin by controlled particle deposition (CPD) using $\mathrm{SC}_{-} \mathrm{CO}_{2}$. J Supercrit Fluid 2007;39(3):435-43.

10. Sultana T, Jung JM, Hong SS, Lee WK, Gal YS. Characteristic profiles of the inclusion complex of omeprazole/peracylated$\beta$-cyclodextrin formed in supercritical carbon dioxide. J Incl Phenom Macro Chem 2012;72(1-2):207-12.

11. Campardelli R, Baldino L, Reverchon E. Supercritical fluids applications in nanomedicine. J Supercrit Fluid 2015;101:193214.

12. Girotra P, Singh SK, Nagpal K. Supercritical fluid technology: a promising approach in pharmaceutical research. Pharm Dev Technol 2013;18(1):22-38.

13. Esfandiari N. Production of micro and nanoparticles of pharmaceutical by supercritical carbon dioxide. J Supercrit Fluid 2015;100:129-41.

14. Jia JF, Song N, Gai Y, Zhang L, Zhao Y. Release-controlled curcumin proliposome produced by ultrasound-assisted supercritical antisolvent method. J Supercrit Fluid 2016;113:150-57.

15. Jia J, Wang J, Zhang K, Zhou D, Ge F. Aescin nanoparticles prepared using SEDS: composition stability and dissolution enhancement. J Supercrit Fluid 2017;130:267-72.

16. Van Hees T, Piel G, de Hassonville SH, Evrard B, Delattre L. Determination of the free/included piroxicam ratio in cyclodextrin complexes: comparison between UV spectrophotometry and differential scanning calorimetry. Eur J Pharm Sci 2002;15(4):347-53.

17. Al-Marzouqui AH, Shehatta I, Jobe B, Dowaidar A. Phase solubility and inclusion complex of itraconazole with $\beta$-cyclodextrin using supercritical carbon dioxide. J Pharm Sci 2006;95(2):292-304.

18. Sauceau M, Rodier E, Fages J. Preparation of inclusion complex of piroxicam with cyclodextrin by using supercritical carbon dioxide. J Supercrit Fluid 2008;47(2):326-32.

19. Marco De I, Reverchon E. Influence of pressure, temperature and concentration on the mechanisms of particle precipitation in supercritical antisolvent micronization. J Supercrit Fluid 2011;58(2):295-302.

20. Reverchon E, Torino E, Dowy S, Braeuer A, Leipertz A. Interactions of phase equilibria, jet fluid dynamics and mass transfer during supercritical antisolvent micronization. Chem Eng J 2010;156(2):446-58.

21. Jia JF, Wang W, Gao Y, Zhao Y. Controlled morphology and size of curcumin using ultrasound in supercritical $\mathrm{CO}_{2}$ antisolvent. Ultrason Sonochem 2015;27:389-94.

22. Yu W, Chen B, Zhao Y, Jiang S. Preparation of Silymain Nanoparticles by SEDS Technique. J Chem Eng Chin Univ 2005;19(5):695-98.

23. Machmudah S. Nanoparticle formation of lycopene / $\beta$ cyclodextrin inclusion complex using supercritical antisolvent precipitation. J Supercrit Fluid 2013;83:97-103.

24. Al-Marzouqi AH, Elwy HM, Shehadi I, Adem A. Physicochemical properties of antifungal drug-cyclodextrin complexes prepared by supercritical carbon dioxide and by conventional techniques. J Pharm Biomed Anal 2009;49(2):227-33. 
www.ijpsonline.com

25. Liu H. Study on changes of the IR absorption bands for the guest molecule in $\beta$-cyclodextrin inclusion complex. Spectrosc Spect Anal 2008;28(10):91-92.
26. Yang Z, Chai K, Ji H. Selective inclusion and separation of cinnamaldehyde and benzaldehyde by insoluble $\beta$-cyclodextrin polymer. Sep Purif Technol 2011;80(2):209-16. 